\title{
Exploiting transport properties for the detection of optical pumping in heavy ions
}

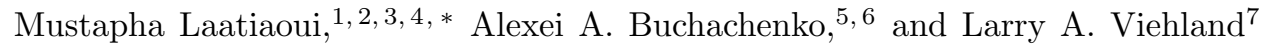 \\ ${ }^{1}$ Department Chemie, Johannes Gutenberg-Universität, \\ Fritz-Strassmann Weg 2, 55128 Mainz, Germany \\ ${ }^{2}$ Helmholtz-Institut Mainz, Staudingerweg 18, 55128 Mainz, Germany \\ ${ }^{3}$ GSI Helmholtzzentrum für Schwerionenforschung GmbH, Planckstrasse 1, D-64291 Darmstadt, Germany \\ ${ }^{4}$ KU Leuven, Instituut voor Kern- en Stralingsfysica, Celestijnenlaan 200D, B-3001 Leuven, Belgium \\ ${ }^{5}$ CEST, Skolkovo Institute of Science and Technology, \\ Skolkovo Innovation Center, Nobel str. 3, Moscow 121205, Russia \\ ${ }^{6}$ Institute of Problems of Chemical Physics RAS, Chernogolovka, Moscow District 142432, Russia \\ ${ }^{7}$ Science Department, Chatham University, Pittsburgh, Pennsylvania 15232, USA
}

(Dated: March 3, 2022)

\begin{abstract}
We present a kinetic model for optical pumping in $\mathrm{Lu}^{+}$and $\mathrm{Lr}^{+}$ions as well as a theoretical approach to calculate the transport properties of $\mathrm{Lu}^{+}$in its ground ${ }^{1} S_{0}$ and metastable ${ }^{3} D_{1}$ states in helium background gas. Calculations of the initial ion state populations, the field and temperature dependence of the mobilities and diffusion coefficients, and the ion arrival time distributions demonstrate that the ground- and metastable-state ions can be collected and discriminated efficiently under realistic macroscopic conditions.
\end{abstract}

\section{INTRODUCTION}

Atomic structure studies complement element discoveries and advance our understanding of atoms and their nuclei [1, 2]. In Ref. [3, a new method of optical spectroscopy is being developed for such studies on element cations beyond nobelium $(Z>102)$, which are currently inaccessible by common techniques. Optical pumping utilizing laser resonant excitations is envisaged to populate metastable electronic states of ions, which can be then discriminated from ions in the ground state utilizing electronic state chromatography [4, 5]. The latter technique exploits the fact that monoatomic ions in different electronic states experience different interactions in ion-atom collisions. In particular, a change in the electronic configuration, such as that encountered when metastable states are occupied, results in different transport coefficients (ion mobility and diffusion coefficients), which control the rate of the field-induced ion drift through an inert gas 6. While the difference in ion-atom interaction reflects the fundamental electronic structures, the drift time of ions is controlled by macroscopic parameters (temperature, pressure, and electricfield strength), which can be optimized to achieve the best collection or discrimination of the ions. The use of electronic state chromatography in conjunction with ablation sources for state-selected ion chemistry is an established technique [7]. Such studies can span a variety of elemental cations along the first-, second-, and third-row transition metals for which state-specific mobilities have already been measured $8-10$. Importantly, in this context, electronic structure information can be extracted while searching for suitable ground-state transitions for resonant optical pumping (see Sec. II).

\footnotetext{
* mlaatiao@uni-mainz.de
}

In the present work, we provide the theoretical proof of the optical spectroscopy concept, called laser resonance chromatography [3], for $\mathrm{Lu}^{+}$and its heavier chemical homologue, $\operatorname{Lr}^{+}(Z=103)$. Following the concept, a few ions are first bunched inside a radiofrequency buncher for optical pumping and then released to a drift tube for electronic-state chromatography. In the following Sec. III. we propose a simple resonant pumping scheme allowing for an efficient population of the metastable states of the ions. We predict the interaction potentials of $\mathrm{Lu}^{+}$in the metastable state with helium in Sec. III and describe the gaseous ion transport in ground and metastable states at different conditions of temperature $T$ and ratio of electric-field strength to gas number density $E / n_{0}$ in Sec. [V] Based on the analysis of expected arrival time distributions introduced in Sec. V w we identify parameter ranges for electronic state chromatography in Sec. VI and deduce achievable ion transmission and collection efficiencies, and the efficiency for detecting resonant optical excitations.

\section{OPTICAL PUMPING IN SINGLY-CHARGED LUTETIUM AND LAWRENCIUM}

We developed a rate equation model for a five-level system to evaluate optical pumping in $\mathrm{Lu}^{+}\left(\mathrm{Lr}^{+}\right)$prior to electronic state chromatography. The system consists of the ground state $|1\rangle: 6 s^{2}{ }^{1} S_{0}\left(7 s^{2}{ }^{1} S_{0}\right)$, the intermediate level $|2\rangle: 6 s 6 p^{3} P_{1}\left(7 s 7 p^{3} P_{1}\right)$ that should be probed by laser radiation, and three low-lying metastable states $|3\rangle: 6 s 5 d^{1} D_{2}\left(7 s 6 d^{1} D_{2}\right),|4\rangle: 6 s 5 d^{3} D_{2}\left(7 s 6 d^{3} D_{2}\right)$, and $|5\rangle: 6 s 5 d^{3} D_{1}\left(7 s 6 d^{3} D_{1}\right)$ [11, 12, which serve to collect the population from $|2\rangle$ by radiative and collision-induced relaxation processes [3] (see inset in Fig. 1). Collisional quenching was considered for ${ }^{3} P_{1}$ to ${ }^{1} D_{2},{ }^{3} D_{2}$ to ${ }^{3} D_{1}$, and ${ }^{3} D_{1}$ to ${ }^{1} S_{0}$ at different gas number densities $n_{0}$ by including the corresponding quenching rates as reported 
for the isoelectronic neutral barium in $\mathrm{He}$ at $880 \mathrm{~K}$, $\alpha_{23} / n_{0}=8 \times 10^{-11} \mathrm{~cm}^{3} / \mathrm{s}, \alpha_{45} / n_{0}=6 \times 10^{-11} \mathrm{~cm}^{3} / \mathrm{s}$, and $\alpha_{51} / n_{0}=10^{-13} \mathrm{~cm}^{3} / \mathrm{s}$, respectively [13]. In addition, it is assumed that broadband laser radiation is used during the initial level search such that the coherence terms in the optical Bloch equations can be safely neglected [14. We obtain

$$
\begin{aligned}
\frac{d \rho_{1}}{d t} & =A_{21} \rho_{2}+A_{31} \rho_{3}+A_{41} \rho_{4}+\left(A_{51}+\alpha_{51}\right) \rho_{5} \\
& -\frac{1}{2} A_{21} S\left(\omega_{L}, \omega_{12}\right) O(t)\left(\rho_{1}-\rho_{2}\right) \\
\frac{d \rho_{2}}{d t} & =\frac{1}{2} A_{21} S\left(\omega_{L}, \omega_{12}\right) O(t)\left(\rho_{1}-\rho_{2}\right) \\
& -\left(A_{21}+A_{23}+A_{24}+A_{25}+\alpha_{23}\right) \rho_{2} \\
\frac{d \rho_{3}}{d t} & =\left(A_{23}+\alpha_{23}\right) \rho_{2} \\
& -\left(A_{31}+A_{34}^{e}+A_{34}^{m}+A_{35}^{e}+A_{35}^{m}\right) \rho_{3} \\
\frac{d \rho_{4}}{d t} & =A_{24} \rho_{2}+\left(A_{34}^{e}+A_{34}^{m}\right) \rho_{3} \\
& -\left(A_{41}+A_{45}^{e}+A_{45}^{m}+\alpha_{45}\right) \rho_{4} \\
\frac{d \rho_{5}}{d t} & =A_{25} \rho_{2}+\left(A_{35}^{e}+A_{35}^{m}\right) \rho_{3} \\
& +\left(A_{45}^{e}+A_{45}^{m}+\alpha_{45}\right) \rho_{4}-\left(A_{51}+\alpha_{51}\right) \rho_{5}
\end{aligned}
$$

with the normalization $\sum_{i} \rho_{i}=1$ and the initial conditions $\rho_{1}(t=0)=1$ and $\rho_{i}(t=0)=0$ for $1<i$, where $\rho_{i}$ with $i=1 . . .5$, correspond to the occupations of individual states $|i\rangle . A_{k i}, A_{k i}^{e}$, and $A_{k i}^{m}$ are the Einstein coefficients for spontaneous emission from $|k\rangle$ to $|i\rangle$ via $E 1, E 2$, and $M 1$ transitions, respectively [11, 12, 15, 16].

We used the frequency-dependent saturation parameter $S\left(\omega_{L}, \omega_{12}\right)$ as described in Ref. [17. In this parameter, we considered Doppler broadening at room temperature in terms of full width at half maximum of $0.8 \mathrm{GHz}$ $(0.7 \mathrm{GHz})$ for $\mathrm{Lu}^{+}\left(\mathrm{Lr}^{+}\right)$and the spectral bandwidth of the laser of $2.5 \mathrm{GHz}$, as well as dephasing effects on the order of $1.4 \mathrm{GHz}$ from mode fluctuations within the laser pulse 17. For both ionic species, an energy density of the laser radiation of $10 \mu \mathrm{J} / \mathrm{cm}^{2}$ was taken. For simplicity's sake, we neglected hyperfine structures and nuclear isomerism as these should be covered by the broadband laser radiation. In addition, we neglected broadening effects from radial macro-motion of ions as well as collisional dephasing effects because they do not affect the results at the expected background pressures. Moreover, we included a rectangular function $O(t)$ into the model to mimic laser pulse exposures of $10 \mathrm{~ns}$ duration and $100 \mu \mathrm{s}$ period.

Figure 1 shows the calculated level occupations in $\mathrm{Lu}^{+}$ in the course of 10 laser exposures at a He background pressure of $5 \times 10^{-2}$ mbar. The efficiency for optical pumping from the ground state into the ${ }^{3} D_{1}$ level is $31.5 \%$ for one laser pulse exposure and reaches a value of $94 \%$ for 10 laser pulses. Similar calculations for $\mathrm{Lr}^{+}$indicate a lower but still sufficiently high efficiency of $7.5 \%$ and $53 \%$ for one and 10 pulse exposures, respectively.

In addition, a substantial population transfer in both

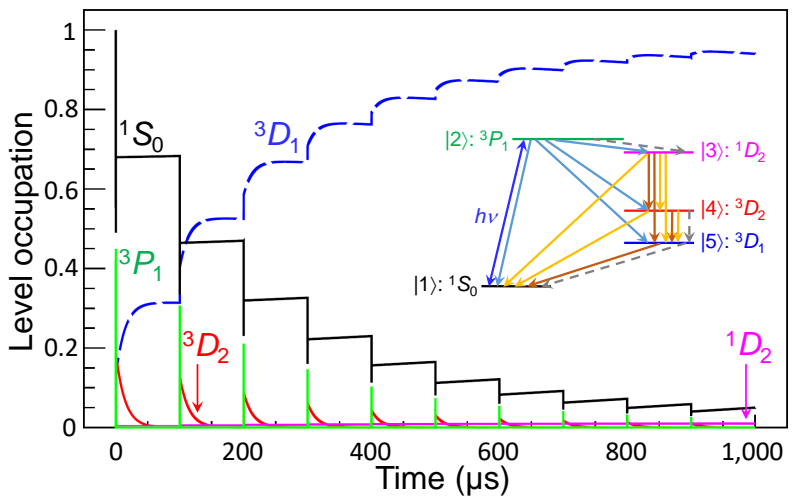

FIG. 1. Laser-induced population transfer from ground- to metastable states in $\mathrm{Lu}^{+}$at $5 \times 10^{-2}$ mbar He. Level occupation is indicated for each of the modeled states $|i\rangle$ in the course of 10 laser beam exposures. Inset: corresponding fivelevel system used in the rate-equation model with arrows in blue, yellow, and brown indicating $E 1, E 2$, and $M 1$ transitions, respectively. Laser probing $(h \nu)$ of the intermediate ${ }^{3} P_{1}$ state induces optical pumping in the system. Collisioninduced relaxation is marked by dashed arrows.

ionic species can be obtained to the ${ }^{1} D_{2}\left({ }^{3} D_{2}\right)$ level at $5 \times 10^{-1}$ mbar $\left(5 \times 10^{-3} \mathrm{mbar}\right)$ background pressures. Collisional quenching is found to depopulate the ${ }^{3} D_{1}$ state only slowly at $5 \times 10^{-1}$ mbar and is a negligibly small effect for pressures $\leq 5 \times 10^{-2}$ mbar.

\section{III. $\mathrm{Lu}^{+}-\mathrm{He}$ INTERACTION POTENTIALS}

The scalar-relativistic (SR) interaction potentials between the ground-state $\mathrm{Lu}^{+}\left(6 s^{2}{ }^{1} S\right)$ ion and rare gas atoms from He to Xe were calculated $a b$ initio in Ref. [18. The reduced zero-field mobility in He at room temperature computed as described in Sec. IV] below is $K_{0}=$ $16.578 \mathrm{~cm}^{2} /$ Vs. This was later confirmed experimentally as $K_{0}=16.8 \pm 0.4 \mathrm{~cm}^{2} / \mathrm{Vs}[19$. Good agreement with the experimental data was also found for other lanthanide ions studied: $\mathrm{Yb}^{+}, \mathrm{Eu}^{+}$and $\mathrm{Gd}^{+}[6,18,20]$. We therefore used similar theoretical techniques to address the interaction of the metastable $\mathrm{Lu}^{+}\left(6 s 5 d^{3} D\right)$ ion with He.

In brief, the small-core effective core potential (ECP) ECP28MWB 21 and the segmented basis set 22] augmented by the $s 2 p d f g$ set of primitive diffuse functions 23. were employed for Lu, whereas the aug-cc-pV5Z basis was used for He [24. The $3 s 3 p 2 d 2 f 1 g$ bond function set 25] was placed in the middle of the $\mathrm{Lu}-\mathrm{He}$ distance. The ground-state $X^{1} \Sigma^{+} \mathrm{SR}$ potential was computed as in Ref. 18 using the restricted coupled-cluster method with singles, doubles, and noniterative triples, $\operatorname{CCSD}(\mathrm{T})$ 26, 27, as implemented in the MOLPRO program package 28. for the restricted Hartree-Fock reference. For the metastable state, the restricted HartreeFock wave functions were calculated by fixing the single occupation of the particular $\mathrm{Lu}^{+} 5 d_{0} \sigma$ or $5 d_{+2} \delta$ molecular orbital, allowing us to resolve ${ }^{3} \Sigma^{+}$and ${ }^{3} \Delta$ molecular 
TABLE I. Equilibrium distances $R_{e}$, well depths $D_{e}$, and dissociation energies $D_{0}$ of the $\mathrm{Lu}^{+}-$He potentials corresponding to the ground ${ }^{1} S\left({ }^{1} S_{0}\right)$ and metastable ${ }^{3} D\left({ }^{3} D_{1}\right)$ states of the ion.

\begin{tabular}{lccc}
\hline \hline State & $R_{e}(\AA)$ & $D_{e}\left(\mathrm{~cm}^{-1}\right)$ & $D_{0}\left(\mathrm{~cm}^{-1}\right)$ \\
\hline \multicolumn{3}{c}{ Scalar relativistic } \\
$X^{1} \Sigma^{+}\left({ }^{1} S\right)$ & 4.17 & 47.3 & 31.4 \\
${ }^{3} \Sigma^{+}\left({ }^{3} D\right)$ & 4.41 & 32.6 & 20.3 \\
${ }^{3} \Pi\left({ }^{3} D\right)$ & 3.77 & 61.0 & 43.9 \\
${ }^{3} \Delta\left({ }^{3} D\right)$ & 3.83 & 62.8 & 45.2 \\
& & SO coupled \\
$X 0^{+}\left({ }^{1} S_{0}\right)$ & 4.17 & 47.3 & 31.4 \\
$0_{1}^{-}\left({ }^{3} D_{1}\right)$ & 4.11 & 49.9 & 34.2 \\
$1_{1}\left({ }^{3} D_{1}\right)$ & 3.91 & 52.2 & 36.4 \\
\hline \hline
\end{tabular}

states that fall in the same $A_{1}$ representation of the $C_{2 v}$ symmetry group. The CCSD $(\mathrm{T})$ method was then implemented for each reference wave function, with the $\mathrm{Lu}^{+}$ $4 s^{2} 4 p^{6} 4 d^{10}$ shells kept as core and the counterpoise correction [29] applied individually to each state. This stateresolved approach was proven to be successful for the $\mathrm{Gd}^{+}\left({ }^{10} D^{\circ}\right)-\mathrm{He}$, Ar interactions [20. TableI presents the equilibrium parameters, distance $R_{e}$ and well depth $D_{e}$, and dissociation energy $D_{0}$ obtained in the SR $\operatorname{CCSD}(\mathrm{T})$ calculations.

To take into account the spin-orbit (SO) coupling that determines the fine structure of the metastable state, the state-interacting SO configuration-interaction method 30] was employed. The ECP description gives poor results for SO coupling [20], so we resorted to the all-electron description. It was found that the measured fine-structure splittings 11 cannot be reproduced well for isolated ${ }^{3} \mathrm{D}$ multiplet. Test calculations indicated that improvement can be achieved by taking into account the higher lying $6 s 5 d^{1} D$ term. The SR potentials correlating to this term were computed using the multireference configuration-interaction method [31, 32] with the state-averaged complete active space multiconfigurational self-consistent field reference orbitals [33] and the ECP approach described above. The resulting SR potentials together with the $\operatorname{CCSD}(\mathrm{T})$ potentials for the ground and metastable triplet states were taken as the diagonal part of the SO Hamiltonian matrix. The BreitPauli coupling matrix elements were calculated adopting the all-electron X2C ("exact two-component") 34 SR approximation with the cc-pwCVDZ and aug-cc-pVDZ basis sets for $\mathrm{Lu}$ and $\mathrm{He}$, respectively 24, 35. In all multireference calculations, $\mathrm{Lu}^{+} 6 s 5 d$ orbitals were considered as active, while the $\mathrm{Lu}^{+} 5 p 4 f$ and $\mathrm{He} 1 s$ orbitals were correlated as doubly occupied.

The resulting energies of the ${ }^{3} D_{J}$ fine-structure levels $J=2,3$ with respect to the one with $J=1$ are 639 and $2236 \mathrm{~cm}^{-1}$, which are in good agreement with the experimental values of 639 and $2403 \mathrm{~cm}^{-1}$ [11, respectively.

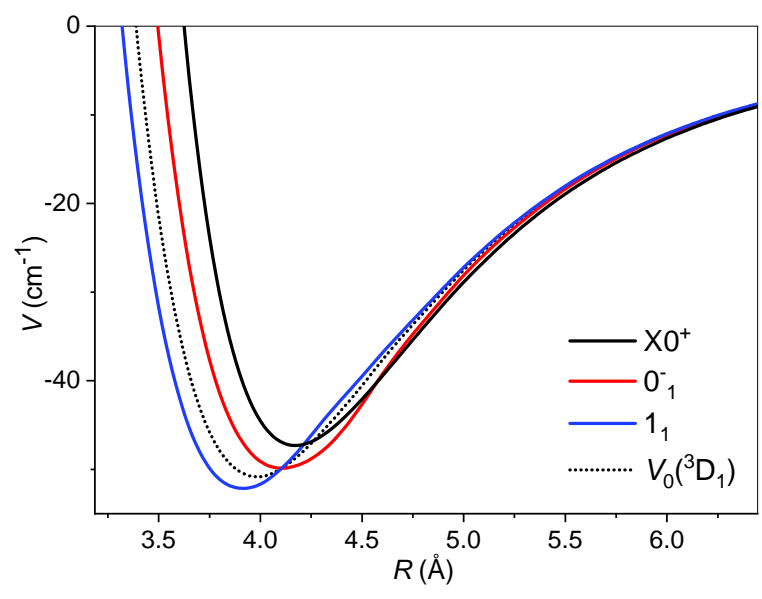

FIG. 2. $\mathrm{Lu}^{+}-\mathrm{He}$ SO-coupled interaction potentials corresponding to the ${ }^{1} S_{0}$ and ${ }^{3} D_{1}$ states of the ion. Dotted line represents isotropic interaction potential for the ${ }^{3} D_{1}$ state. For the sake of comparison, all potentials are referred to the same dissociation limit.

The SO-coupled potentials relevant to the transport calculations are shown in Fig. 2, while their parameters are presented in Table I. In the $\Omega_{J}^{\sigma}$ notations of the Hund case (c) coupling scheme, with $J$ and $\Omega$ being the total angular electronic momentum and its projection onto the interatomic axis, respectively, and $\sigma$ being inversion parity, these states are $X 0^{+}$, which replaces the ground $X^{1} \Sigma^{+}$SR state, and $0_{1}^{-}, 1_{1}$, which correlate to the ${ }^{3} D_{1}$ $\mathrm{Lu}^{+}$term. One should note that the He interaction with the metastable $\mathrm{Lu}^{+}$ion is stronger than that with the ground-state ion.

\section{TRANSPORT COEFFICIENTS}

Transport properties were calculated for the ${ }^{175} \mathrm{Lu}^{+}$ ion using the Gram-Charlier approach to solving the Boltzmann equation [36 38. For the ground-state ion, the momentum-transfer and other transport cross sections were calculated as functions of the collision energy for the single $X 0^{+}$interaction potential. For the metastable $\mathrm{Lu}^{+}\left({ }^{3} D_{1}\right)$ ion, we used so-called "anisotropic SO-coupled approximation" [18, 20, 38, in which the cross sections for the $0_{1}^{-}$and $1_{1}$ interaction potentials were averaged with the degeneracy factors $1 / 3$ and $2 / 3$, respectively.

Figure 3 shows the temperature dependence of the reduced zero-field mobilities for the ions in two electronic states, while the reduced mobility dependence on the ratio of the field strength to gas number density is presented in Fig. 4 Effects of temperature and $E / n_{0}$ are quite remarkable. With increasing $T$, deviations in zero-field mobilities of up to $24 \%$ can be reached. A so-called low-field region with constant mobility holds below $E / n_{0} \approx 10 \mathrm{Td}$, with $1 \mathrm{Td}$ (Townsend unit) being equal to $10^{-21} \mathrm{Vm}^{2}$; 


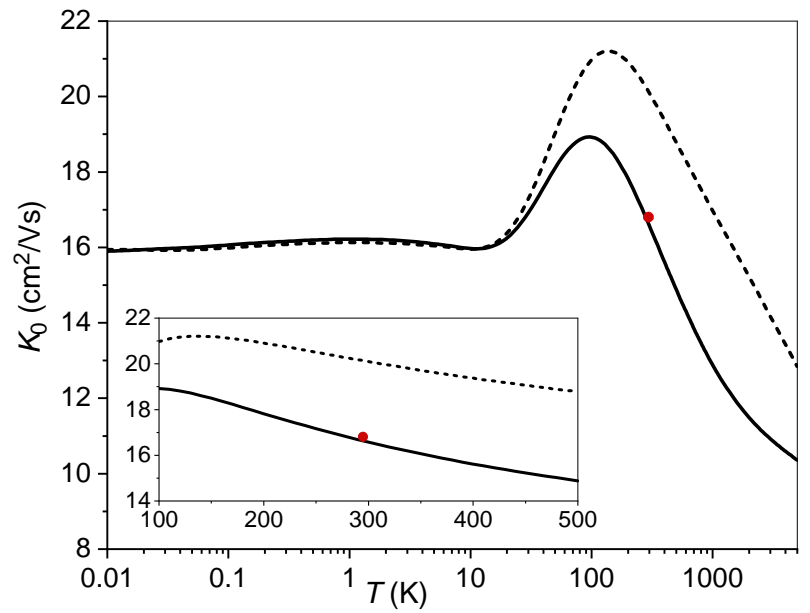

FIG. 3. Reduced zero-field mobilities of the $\mathrm{Lu}^{+}$ions in the ground $\mathrm{X0}^{+}$state (solid line) and metastable ${ }^{3} D_{1}$ state (dashed line) as functions of temperature. The dot indicates the experimental result for the ground state 19. The inset provides an enlarged view of the $100-500 \mathrm{~K}$ region.

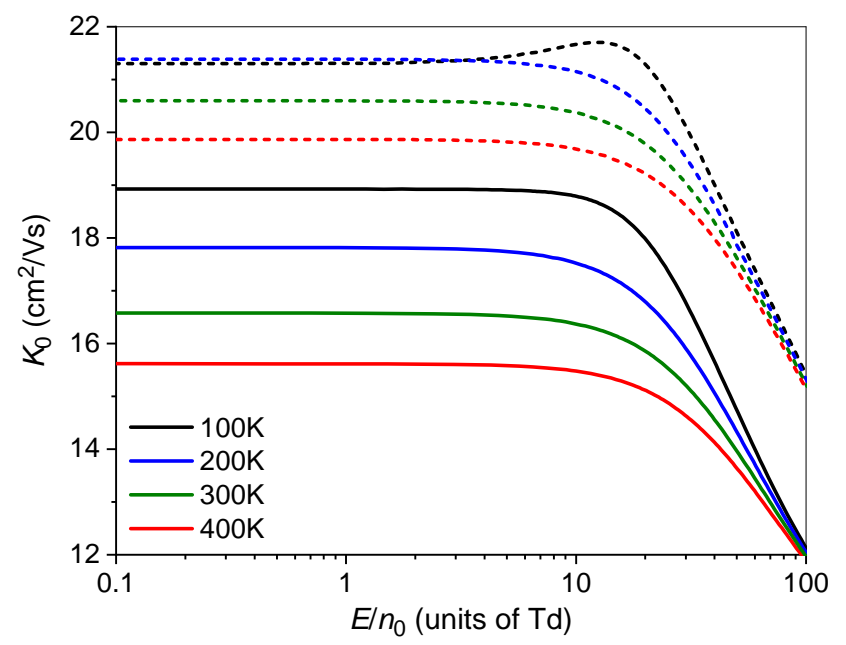

FIG. 4. Reduced mobilities of the $\mathrm{Lu}^{+}$ions in the ground $\mathrm{XO}^{+}$state (solid lines) and metastable ${ }^{3} D_{1}$ state (dashed lines) as functions of $E / n_{0}$ at selected temperatures.

then the mobilities of ions in both states start to decline.

We also calculated the diffusion coefficients, as ion diffusion leads to ion losses and unwanted broadening of drift time distributions. Zero-field values of the $n_{0} D$ products are depicted in Fig. 5 as functions of temperature. Lower temperatures minimize both absolute diffusion effects and their difference for two electronic states. An external electrostatic field distinguishes longitudinal diffusion along the field direction and transverse diffusion perpendicular to it. The rapid increase with $E / n_{0}$ of the longitudinal diffusion coefficients $D_{L}$ multiplied by $n_{0}$ is shown in Fig. 6. The transverse coefficients $D_{T}$ behave similarly. Similar to mobility, strong variation of the dif-

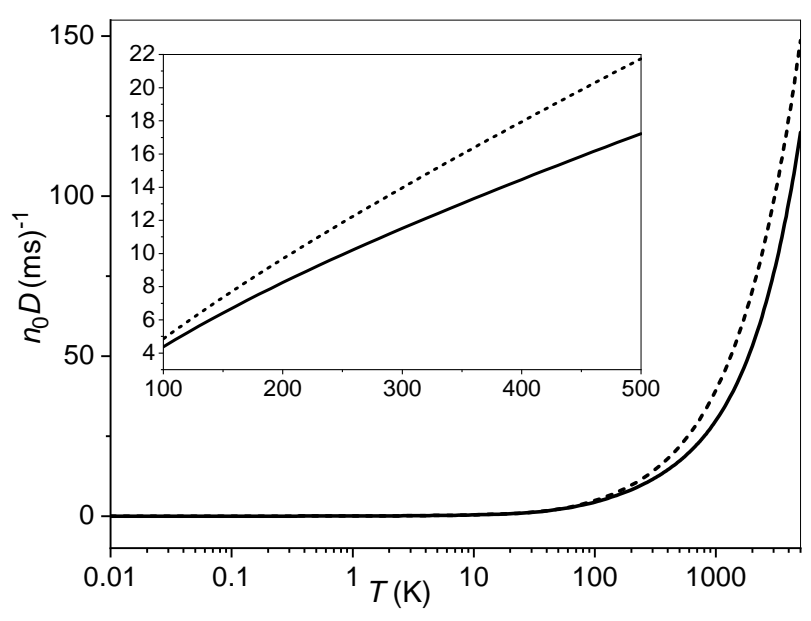

FIG. 5. Zero-field diffusion coefficients $n_{0} D$ of the $\mathrm{Lu}^{+}$ions in the ground $X 0^{+}$state (solid line) and metastable ${ }^{3} D_{1}$ state (dashed line) as functions of temperature. Inset provides an enlarged view of the $100-500 \mathrm{~K}$ region.

fusion coefficients takes place at $E / n_{0}>10 \mathrm{Td}$.

From these transport coefficient calculations we conclude that metastable-state ions drift faster than the ground-state ones, but experience more diffusion.

Another important parameter is the effective kinetic temperature of the ion $T_{\text {eff }}$ 37. Being related to the transport coefficients, $T_{\text {eff }}$ defines the kinetic energy of the ion-atom collision and hence affects the rate of collision-induced quenching of excited states. We found that this stays close to buffer gas temperature $T$ up to $E / n_{0} \approx 10 \mathrm{Td}$ and then rapidly increases; see Fig. 7 .

In order to achieve the best discrimination of ions in the metastable state a detailed analysis of the drift times is in order. Bearing in mind the pumping model introduced before wherein quenching rates for $\mathrm{Ba}$ at $880 \mathrm{~K}$ were used, we considered $E / n_{0}$ values up to $40 \mathrm{Td}$ in our analysis in Sec. VI to guarantee that $T_{\text {eff }}$ stays below $1100 \mathrm{~K}$.

\section{DRIFT TIME ANALYSIS}

Ion mobility $K$ determines the steady-state drift velocity of an ion in a buffer-gas filled drift tube under the influence of a permanent electric field $E$ according to $v_{d}=K E$. The mean time needed for an ion swarm to pass through the tube is

$$
t_{d}=\frac{L}{K E}=\frac{L}{N_{L} K_{0}\left(E / n_{0}\right)},
$$

where the definition of reduced mobility $K_{0}=n_{0} K / N_{L}$ is used and $N_{L}$ is the Loschmidt number. The drift time depends on the drift length $L$, the gas temperature $T$ (through $K_{0}$ and $n_{0}$ ), the pressure $p_{0}\left(\right.$ through $\left.n_{0}\right)$, and 


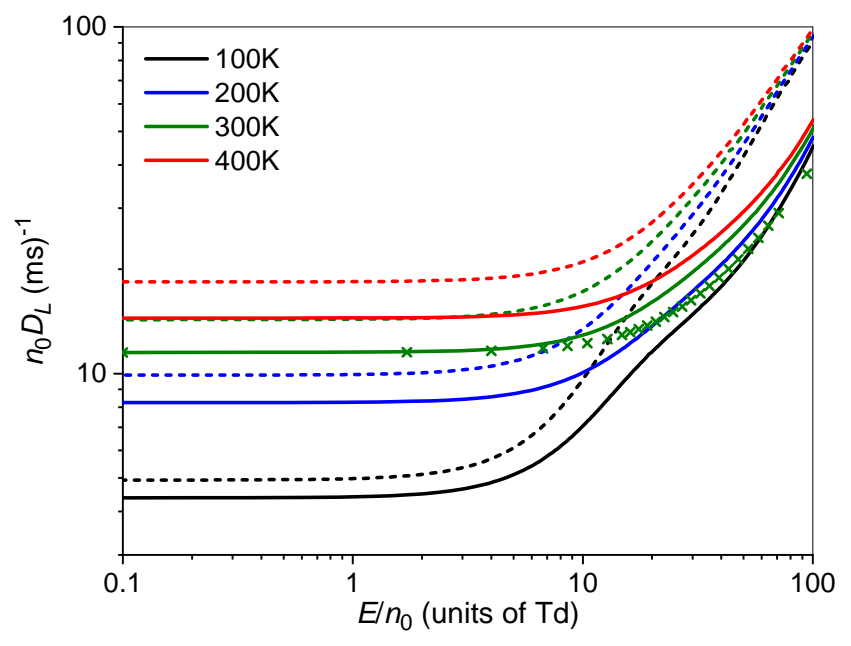

FIG. 6. Longitudinal diffusion coefficients $n_{0} D_{L}$ of the $\mathrm{Lu}^{+}$ ions in the ground $X 0^{+}$state (solid lines) and metastable ${ }^{3} D_{1}$ state (dashed lines) as functions of $E / n_{0}$ at selected temperatures. Crosses exemplify the dependence of the transverse coefficient $n_{0} D_{T}$ of the ground-state ion at $T=300 \mathrm{~K}$.

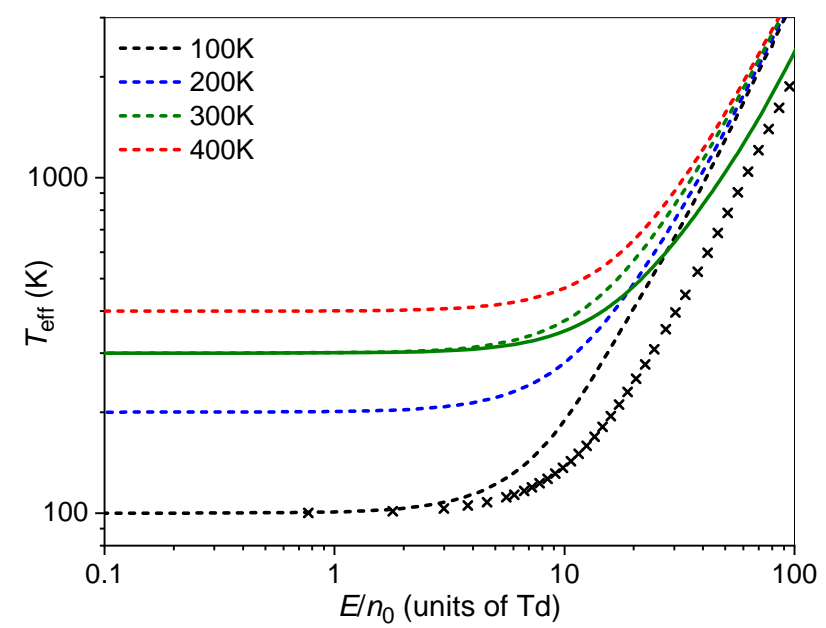

FIG. 7. Effective kinetic temperatures $T_{\text {eff }}$ of the $\mathrm{Lu}^{+}\left({ }^{3} D_{1}\right)$ ions along the drift field at selected buffer gas temperatures as functions of $E / n_{0}$ (dashed lines). Solid line exemplifies the same quantity for the ground-state $\mathrm{Lu}^{+}$ion at $300 \mathrm{~K}$, while crosses show the dependence of $T_{\text {eff }}$ in the direction perpendicular to the field axis at $100 \mathrm{~K}$.

$E / n_{0}$ (explicitly and through $K_{0}$ ). The difference between the drift times of the ground-state ions, $t_{d}$, and of the metastable-state ions, $t_{d}^{*}$, that underlies the electronic state chromatography effect [7] reflects the difference in the respective mobilities as functions of $T$ and $E / n_{0}$. It can be expressed as

$$
\Delta t_{d}=t_{d}-t_{d}^{*}=\frac{L}{N_{L}\left(E / n_{0}\right)} \frac{K_{0}^{*}-K_{0}}{K_{0}^{*} K_{0}} .
$$

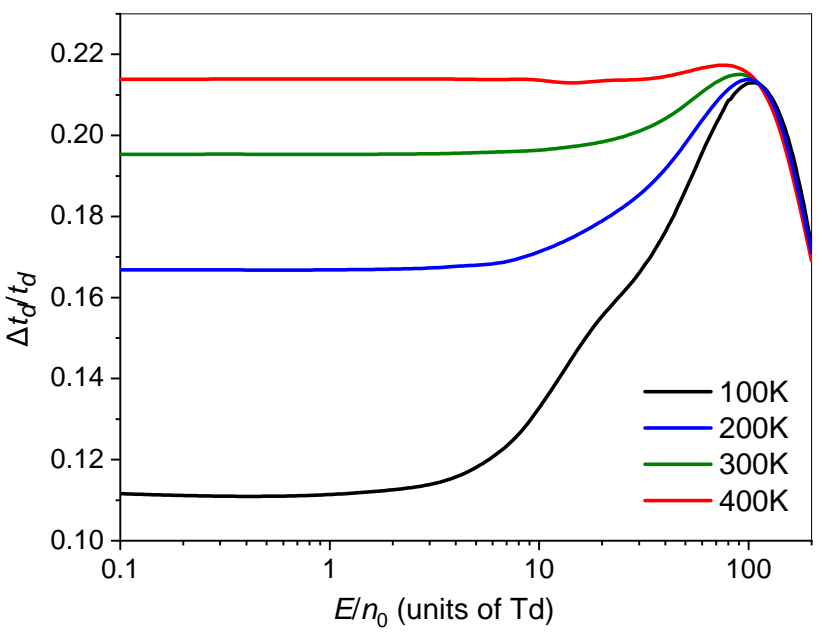

FIG. 8. Relative difference of the drift times for $\mathrm{Lu}^{+}$ions in the ground and metastable states.

More convenient in the present context is to use the reduced time $t / t_{d}$, so that

$$
\Delta t_{d} / t_{d}=\left(K_{0}^{*}-K_{0}\right) / K_{0}^{*}
$$

gives the relative difference of the drift times. This relative difference can be optimized for best chromatography performance by finding proper drift conditions of temperature, pressure, and electric-field strength. The larger the difference the more likely is detection of resonant pumping from the appearance of a distinct metastable-state peak in the arrival time distribution. Figure 8 shows this relative difference at different $T$ as a function of $E / n_{0}$. With rising temperature, $\Delta t_{d} / t_{d}$ gradually increases in the low-field region and loses the dependence on $E / n_{0}$. The maximum difference of about $21 \%$ is achieved either at temperatures above $400 \mathrm{~K}$ for a wide range of $E / n_{0}$ or for $80 \lesssim E / n_{0} \lesssim 100 \mathrm{Td}$. Thus, elevated temperatures help to achieve better discrimination of ions of two types by their mean drift times. However, maximizing this difference is by far not the only prerequisite for reliable detection of the laser resonance. Although lowering the temperature down to $100 \mathrm{~K}$ and thus reducing the resolution down to a minimum of about $11 \%$, one gains an additional degree of freedom through $\Delta t_{d} / t_{d}$ dependence on $E / n_{0}$ and a substantial reduction in diffusion and in effective temperature of the ion.

The mean drift time characterizes the center of the distribution created by the ion swarm arriving to a detector. The shape of the distribution depends on the diffusional spread of the swarm along the field axis, while diffusion in a plane perpendicular to the field causes ion losses. For more realistic simulations of these factors we used the analytical expression for the ion flux derived by Moseley et al. [39] (see also Refs. [37] and 40]). The swarm of ions is assumed to be injected instantaneously from the buncher at time $t=0$ into the cylindrical drift tube along its axis that coincides with the external electricfield direction. This generates an ion flux at a distance $z$ 
of

$$
\begin{aligned}
f(z, t) & =\frac{b}{4 \pi^{3 / 2} r_{2}^{2}} \frac{z+v_{d} t}{D_{L}^{1 / 2} t^{3 / 2}} \exp \left[-\frac{\left(z-v_{d} t\right)^{2}}{4 D_{L} t}\right] \\
& \times\left[1-\exp \left(-\frac{r_{1}^{2}}{4 D_{T} t}\right)\right],
\end{aligned}
$$

where $b$ is the number of ions in the swarm. In what follows, we assume $b=1$ and use arbitrary units for the ion flux. In addition to transport coefficients $D_{L}, D_{T}$, and $K_{0}$, Eq. (9) contains the entrance and exit orifice radii, $r_{1}$ and $r_{2}$. The drift tube dimensions are assumed to be much larger than these radii such that Eq. (9) still holds. This equation disregards initial swarm spreads in position and velocity, boundary field imperfections and drift of injected ions before equilibration. However, we expect these minor effects to be similar for ions in different electronic states and unlikely to affect the relative ion counts.

We first consider the principle way to discriminate $\mathrm{Lu}^{+}$ ions in the ground and metastable states. As starting parameters, we chose $r_{1}=r_{2}=1 \mathrm{~mm}, L=6 \mathrm{~cm}$ and a pressure of 1 mbar following Iceman et al. [10], who successfully discriminated metastable states of $\mathrm{Mo}^{+}$and $\mathrm{W}^{+}$ions. For this choice, we calculated the drift time distributions at the distance $z=L$ and $T=300 \mathrm{~K}$ for each of the ion states. Figure 9 (a) shows the contour plot of the $f\left(L, t / t_{d}\right)$ distributions assuming a $1: 1$ abundance of $\mathrm{Lu}^{+}$ions in ground and metastable states depending on $E / n_{0}$ for the initial parameter choice. The distributions are normalized to unity at the highest maximum and thus disregard ion losses. At $5 \mathrm{Td}$ the groundand metastable-state ions cannot be discriminated and jointly form a structureless distribution. As ions move faster with increasing $E / n_{0}$ values, peak broadening decreases and ions in the different states can be discriminated, which is the case for $5<E / n_{0}<200 \mathrm{Td}$. Figure 9 (b) shows $t / t_{d}$ distributions at selected $E / n_{0}$ values where two peaks are clearly visible: one (the "ground peak," $t / t_{d}=1$ ) corresponds to the ground state and another (the "metastable peak," $t / t_{d} \approx 0.8$ ) corresponds to the metastable-state ions. A best peak discrimination is achieved at $E / n_{0}=70 \mathrm{Td}$. However, the difference in ion mobility becomes smaller with increasing $E / n_{0}$ values (cf. Fig. 4). Above $100 \mathrm{Td}$ the mean drift times $t_{d}$ and $t_{d}^{*}$ become very close to each other. In addition, longitudinal diffusion strongly increases in accordance with Fig. 6 and smooths the distribution even though mobilities of the ground- and metastable-state ions differ significantly.

This preliminary consideration indicates that the ground- and metastable-state ions can be readily discriminated at room temperature. In view of expected quenching of the metastable state at elevated $T_{\text {eff }}$, an optimum discrimination is desirable at $E / n_{0}$ values below $40 \mathrm{Td}$. In this case, a reduction of diffusional spread can be achieved by shortening the drift length, lowering the temperature (reducing diffusion coefficients), and/or raising the pressure.

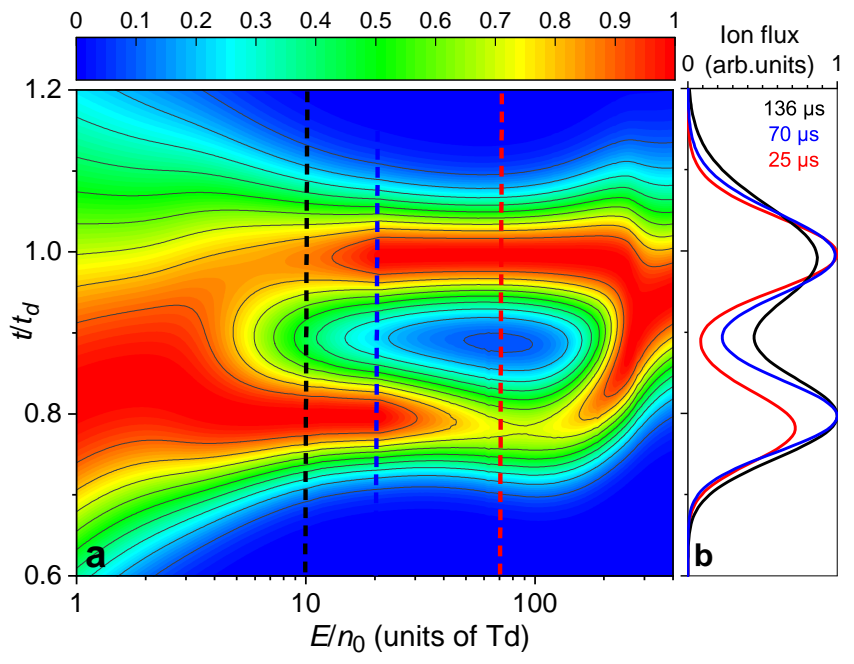

FIG. 9. (a) Relative drift time distribution (color coded) as function of $t / t_{d}$ and $E / n_{0}$ for equal initial ensembles of $\mathrm{Lu}^{+}$ in ground and metastable states. Ion losses are neglected. Horizontal lines mark $E / n_{0}$ sections depicted in panel (b). Drift length is $6 \mathrm{~cm}$, temperature is $300 \mathrm{~K}$, and pressure is 1 mbar. (b) The relative ion fluxes at selected $E / n_{0}$ values of 10 (black), 20 (blue), and $70 \mathrm{Td}$ (red). Legend specifies the corresponding $t_{d}$ values for the ground-state ions.

For a limited number of ions to be investigated as proposed in Ref. 3, ion losses are the next major issue. The diffusional spread of the ion swarm in a direction perpendicular to the electric-field axis strongly affects the efficiency for transmitting ions through the drift tube. Diffusional spread is present for ions in both electronic states, being more significant for the metastable one due to a larger diffusion coefficient; see Fig. 6. This is evident from Fig. 9(b), which shows a decrease of the metastable peak intensity with increasing $E / n_{0}$. Based on Eq. (9), we calculated the transmission efficiency for the metastable $\mathrm{Lu}^{+}\left({ }^{3} D_{1}\right)$ ions as a function of the drift length. As Eq. (9) does not provide normalized ion count, we introduced, following Moseley et al. 39, the timeintegrated flux

$$
A(z)=\int_{0}^{\infty} f(z, t) d t
$$

and defined the transmission efficiency as $A(L) / A(z=$ $1 \mathrm{~mm}$ ) at certain $L, E / n_{0}$ and $p_{0}$ values. The results are presented in Fig. 10 as a contour map of efficiency in the $\left(L, p_{0}\right)$ parameter space at 100,200 and $300 \mathrm{~K}$ and $E / n_{0}$ of $20 \mathrm{Td}$. As expected, for a fixed drift path, buffer gas pressure reduces diffusional losses, but the effect of temperature is much more profound. Cooling the gas down to $100 \mathrm{~K}$ allows one to achieve $90 \%$ transmission in a wide range of pressures. We also infer that the drift length of $L=6 \mathrm{~cm}$ is too long to maintain ion losses below $10 \%$ even at elevated pressures. In general, the shorter the drift length, the smaller the ion losses. The limit is set by the technical feasibility and handling and 


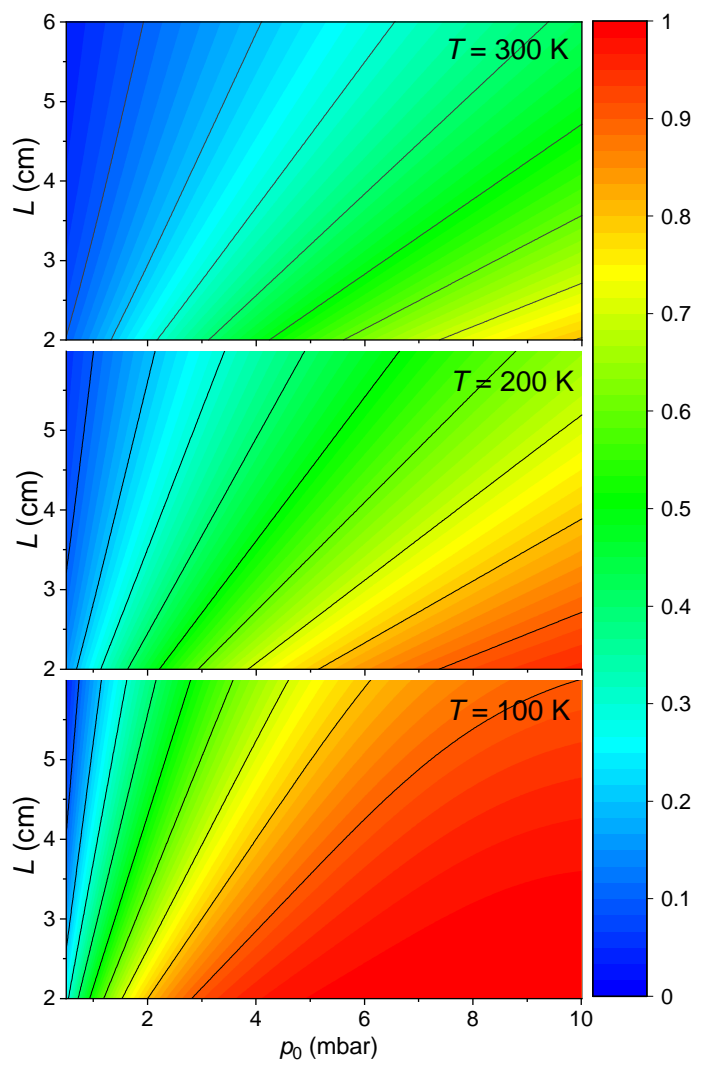

FIG. 10. Transmission efficiency of the $\mathrm{Lu}^{+}\left({ }^{3} D_{1}\right)$ ions (color coded) as function of $L$ and $p_{0}$ at selected temperatures. $E / n_{0}=20 \mathrm{Td}$.

by the initial spatial spread of ions, which could be magnified due to boundary imperfections of the electric-field strength and the nonequilibrated ion motion after injection into the drift tube. A drift length of $L=4 \mathrm{~cm}$ appears a reasonable compromise, allowing one to transmit more than $50 \%$ of the metastable ions at $100 \mathrm{~K}$ and pressures above 1.5 mbar. We also stress that suppressing diffusional losses simultaneously reduces the diffusional spread, hence improving the discrimination of the ions.

The collection of the metastable ions is also affected by collisional quenching during the drift. To estimate the quenching effect conservatively, we repeated the simulations assuming that the quenched metastable ions are lost. This was accounted for by multiplying Eq. (9) by the factor of $\exp \left(-\alpha_{51} t\right)$, where $\alpha_{51}=k n_{0}$ and $k$ is the quenching rate constant [39]. For $k$ we used the value of $10^{-13} \mathrm{~cm}^{3} / \mathrm{s}$, as determined by Brust and Gallagher [13] for the metastable $\mathrm{Ba}\left({ }^{3} D_{1}\right)$ atoms in $\mathrm{He}$ at $880 \mathrm{~K}$; see Sec. III

The efficiency of metastable ion collection, defined with the same normalization as the efficiency of ion transmission, is shown in Fig. 11. Comparison with Fig. 10 illustrates the very significant effect of quenching. At low pressures, the losses are still controlled by diffusion. As the number of ion-atom collisions increases with pressure, quenching losses start to dominate. The interplay

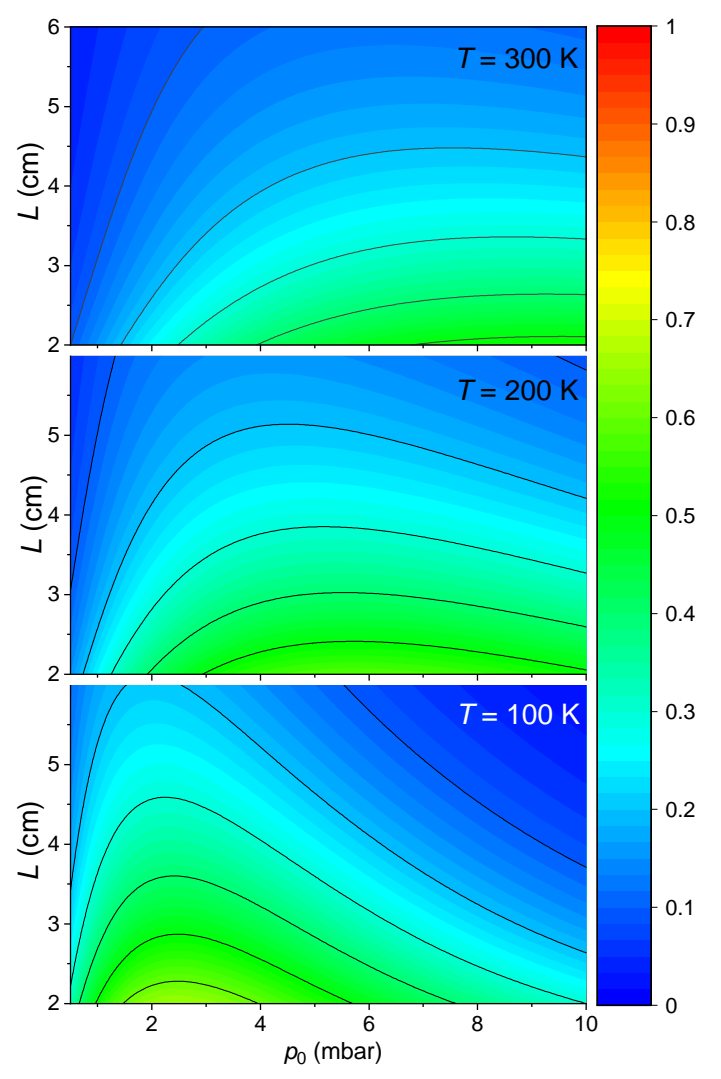

FIG. 11. Collection efficiency of the $\mathrm{Lu}^{+}\left({ }^{3} D_{1}\right)$ ions (color coded) as function of $L$ and $p_{0}$ at selected temperatures. $E / n_{0}=20 \mathrm{Td}$.

between the two effects determines the optimal pressure range. For $T=100 \mathrm{~K}$ and $L=4 \mathrm{~cm}$, for instance, $35 \%$ of the metastable ions can be collected at the exit orifice of the drift tube at pressures between 1.5 and 4 mbar.

\section{RESONANCE DETECTION BY ION COUNT}

In the simulations presented above we identify the range of experimental conditions at which the ions in the metastable states can be discriminated with minimum losses. Accurate simulation of the ion count signal to be detected would require explicit consideration of collisional coupling between the electronic states of ions during the drift. It goes beyond the present analytical picture as the equilibrium between two states is achieved at much longer times than the drift itself. Iinuma and co-workers considered the drift of ions coupled by fast reversible reactions (see Ref. 41] and references therein) but this approach is inappropriate for slow irreversible quenching and reduces to ground-state ions only. We therefore assume the quenching to proceed too slowly to affect the drift time of ions in both states and can only alter their amount by increasing the number of the ground-state ions to the same extent as it decreases the 


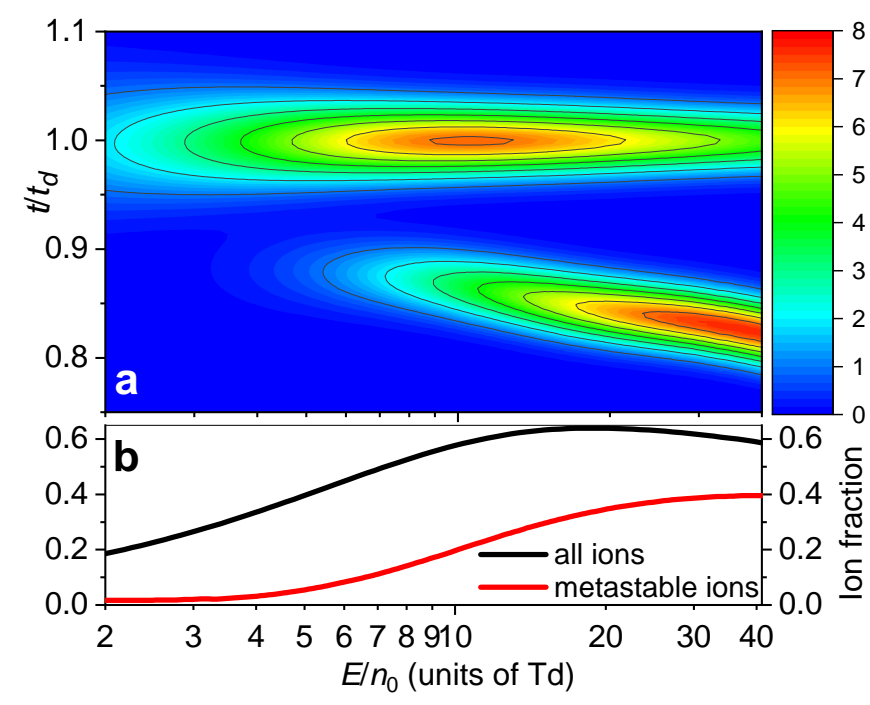

FIG. 12. (a) Ion signal at resonant pumping $I_{p}(t)$ (color coded, arbitrary scale) as function of $t / t_{d}$ and $E / n_{0}$. (b) The fractions of collected ions, all and metastable. For both panels, $T=100 \mathrm{~K}$ and $p_{0}=2 \mathrm{mbar}$.

number of the metastable ions. The flux of the ions in the metastable state is therefore represented by Eq. (9) multiplied by $C_{m} \exp \left(-\alpha_{51} t\right)$, where $C_{m}$ is the initial fraction of the metastable ions in the swarm. The same equation multiplied by $\left[1-\exp \left(-\alpha_{51} t\right)\right] C_{m}+\left(1-C_{m}\right)$ represents the flux of the ground-state ions.

With these weighted functions it is now possible to model expected ion signals with off-resonance and resonant pumping proceeding via $\mathrm{Lu}^{+}{ }^{1} S_{0}{ }^{3} P_{1}$ excitations. We define the former as $I_{g}(t)=f_{g}(L, t)$, where $f_{g}$ is calculated for the swarm of the ground-state ions and $L=$ $4 \mathrm{~cm}$. The signal at resonant pumping $I_{p}(t)=f_{p}(L, t)$ is represented through the combined flux function that accounts for initial ground and metastable state populations of the ions released from the buncher, as well as the population loss and gain due to quenching in the course of the drift. As follows from the population kinetics in the buncher with the residual He pressure of $5 \times 10^{-2}$ mbar (see Sec. II ), $94 \%$ of the ground-state population in $\mathrm{Lu}^{+}$ is transferred to the ${ }^{3} D_{1}$ metastable state at resonance after ten laser pulse exposures. Accordingly, we simulated the signals $I_{g}(t)$ and $I_{p}(t)$ by taking $C_{m}=0.94$. A small ${ }^{1} D_{2}$ occupation fraction predicted by the kinetic model was added to the ground-state population even though its feeding of the lower-lying ${ }^{3} D_{J}$ metastable states is more likely due to efficient intramultiplet collisional relaxation 13. Quenching of metastable states due to ionion interactions has been neglected in view of small ion quantities to be expected in a single bunch. Both signals are normalized to the initial integrated ion flux $A_{0}$ taken at $z=1 \mathrm{~mm}$ according to Eq. $10 \mathrm{p}$ for the off-resonance flux and thus represent the corresponding fractional ion counts.

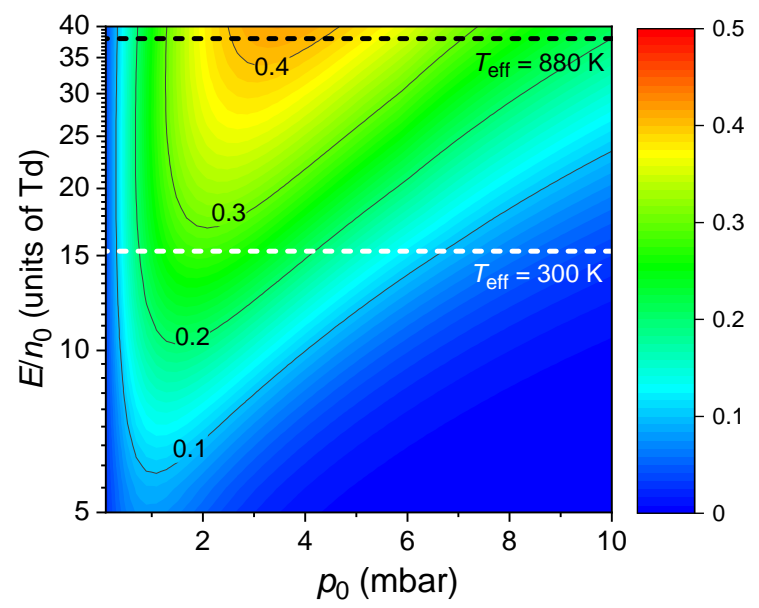

FIG. 13. Fraction of the metastable ions collected after the drift as function of $p_{0}$ and $E / n_{0}$ at $T=100 \mathrm{~K}$. Horizontal lines indicate $E / n_{0}$ values, which correspond to effective temperatures of the metastable ions of 300 and $880 \mathrm{~K}$.

Figure 12(a) shows the signal for resonant pumping $I_{p}(t)$ at $T=100 \mathrm{~K}$ and $p_{0}=2$ mbar as a function of $E / n_{0}$. Though the signal intensities in the $t / t_{d}-E / n_{0}$ map are not representative, the signal evolution is transparent. At low $E / n_{0}$ almost all the metastable ions are lost due to the transverse diffusion and quenching. Despite their minority, the ground-state ions with $t / t_{d}=1$ are still transmitted, having smaller diffusion coefficient and gaining population from quenching. The metastable ions appear at $E / n_{0} \approx 5 \mathrm{Td}$, when dragging force overpowers diffusional spread and makes the quenching probability smaller. Their signal is very well separated in time and grows with $E / n_{0}$. At about $20 \mathrm{Td}$, diffusion losses start to manifest themselves again, as the diffusion coefficients rapidly increase; see Fig. 6. The fraction of collected metastable ions is determined by integrating the $I_{p}(t)$ signal from $t=0$ to $\left(t_{d}+t_{d}^{*}\right) / 2$ and is shown in Fig. 12(b) together with the fraction of all ions detected.

Dependence of the fraction of the metastable ions on both $p_{0}$ and $E / n_{0}$ is mapped in Fig. 13 . Increasing the latter and tuning the pressure to optimum, one can reach a collection efficiency far above $40 \%$. However, increased ion heating from the application of elevated $E / n_{0}$ ratios may promote quenching to the ground state. By constraining the effective ion temperatures below $880 \mathrm{~K}$, we predict a highest collection efficiency of about $41 \%$ at $E / n_{0}=38 \mathrm{Td}$ and $p_{0}=3.5$ mbar. A safer presumption of $300 \mathrm{~K}$ effective temperature results in a collection efficiency of $28 \%\left(E / n_{0} \approx 15 \mathrm{Td}, p_{0} \approx 2 \mathrm{mbar}\right)$.

The expected signals at these conditions are presented in Fig. 14. This shows a complete separation of the peaks for the ground and metastable ions, as well as a reasonably high collection efficiency. It should be noted that a more practical way to detect resonant pumping is to use 


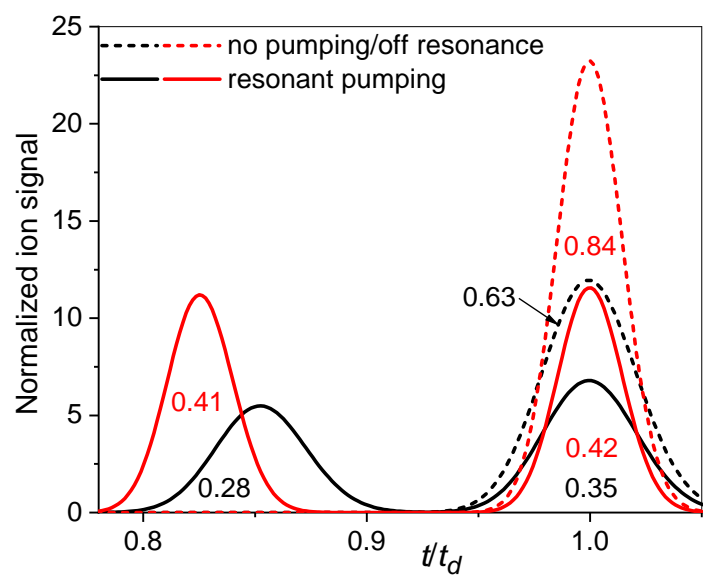

FIG. 14. Off resonance and resonant pumping signals $I_{g}$ and $I_{p}$, respectively, at optimum drift conditions and effective temperatures of metastable ions of $880 \mathrm{~K}$ (red, $p_{0}=3.5 \mathrm{mbar}$, $E / n_{0}=38 \mathrm{Td}$ ) and $300 \mathrm{~K}$ (black, $p_{0}=2 \mathrm{mbar}, E / n_{0}=$ $15 \mathrm{Td}) . L=4 \mathrm{~cm}$ and the He temperature is $T=100 \mathrm{~K}$. Indicated numbers are the respective fractions of ions collected after the drift (areas of the peaks multiplied by $t_{d}$ ).

the difference signal

$$
\Delta I(t)=\frac{1}{2}\left[I_{p}(t)-I_{g}(t)\right] / A_{0},
$$

corresponding to a resonance detection efficiency, where the factor $1 / 2$ accounts for the fact that the difference signal is formally taken from two ion bunches. For the drift time distribution shown in Fig[14, it does not provide any gain in sensitivity, in general. But, given stable experimental conditions while searching for the resonance, the reference signal is collected numerous times such that the difference signal becomes more sensitive to changes in the arrival time distributions, i.e., the efficiency doubles by neglecting $1 / 2$ in Eq. 11.

As discussed in Sec. V] quenching to the ground state significantly impacts the collection efficiency of metastable ions. Since the quenching is related to crossings of potential-energy curves [7, 13, we expect this rate to be smaller and the metastable ion collection to be larger for $\mathrm{Lu}^{+}$drifting in He due to the larger gap in $\mathrm{Lu}^{+}\left(11796 \mathrm{~cm}^{-1}\right)$ compared with $\mathrm{Ba}\left(9034 \mathrm{~cm}^{-1}\right)$.

For a $\mathrm{Lr}^{+}-\mathrm{He}$ system, we expect quenching due to coupling of the potential-energy curves to be much more suppressed, in particular, at low gas temperatures and $E / n_{0}$ ratios because (unlike in $\mathrm{Lu}^{+}$and neutral $\mathrm{Ba}$ ) the ${ }^{3} D_{1}$ state in $\mathrm{Lr}^{+}$is predicted to be far above the ground state $\left(20846 \mathrm{~cm}^{-1}\right)$ 12.

Nevertheless, if we assume that the case of lawrencium differs only in pumping kinetics, the resonant pumping signal from the metastable $\mathrm{Lr}^{+}$ions would be weaker by the initial population ratio of $C_{m}\left(\mathrm{Lr}^{+}\right) / C_{m}\left(\mathrm{Lu}^{+}\right)=$ 0.56 ; see Sec. III. A collection efficiency between $16 \%$ and $23 \%$ can then be expected at optimal conditions, which is sufficiently high for the envisaged studies.

Although other ionic species may exhibit ground-state fine structures compared with $\mathrm{Lu}^{+}$and $\mathrm{Lr}^{+}$, we expect optical pumping to remain efficient in these systems due to intramultiplet collisional relaxation. Similar considerations apply for dark hyperfine components of ground states, which we expect to not play a significant role as power broadening of spectral lines is often beneficial and intended during level searches utilizing broadband lasers.

\section{SUMMARY AND CONCLUSION}

We have modeled optical pumping in $\mathrm{Lu}^{+}$and $\mathrm{Lr}^{+}$ ions and calculated the interaction potentials for $\mathrm{Lu}^{+}$in its ground $\left({ }^{1} S_{0}\right)$ and excited $\left({ }^{3} D_{1}\right)$ state in He. We predict the mobility to be distinct for the two ionic states at temperatures $\gtrsim 100 \mathrm{~K}$, which can be exploited for electronic state chromatography. These calculations and the description of ion drift have enabled us to elucidate parameter spaces of $\left(T, E / n_{0}\right),\left(L, p_{0}\right)$, and $\left(E / n_{0}, p_{0}\right)$ for the best chromatography performance. We found that optical pumping from the ground state would lead to a relatively high collection efficiency of ions in the ${ }^{3} D_{1}$ state at optimal conditions. We expect a state-specific control of the ion transport to be feasible for both ions, $\mathrm{Lu}^{+}$and $\mathrm{Lr}^{+}$. Its usage in conjunction with resonant laser excitations, can be exploited to study the electronic structure of $\mathrm{Lr}^{+}$for which so far only theoretical predictions exist.

\section{ACKNOWLEDGMENTS}

This project has received funding from the European Research Council (ERC) under the European Union's Horizon 2020 research and innovation programme (Grant Agreement No. 819957). A.A.B. acknowledges the support from the Russian Foundation for Basic Research (Project No. 19-03-00144).
[1] K. Blaum, J. Dilling, and W. Nörtershäuser, Precision atomic physics techniques for nuclear physics with radioactive beams, Phys. Scr. T 152, 014017 (2013).

[2] P. Campbell, I. D. Moore, and M. R. Pearson, Laser spectroscopy for nuclear structure physics, Prog. Part. Nucl. Phys. 86, 127 (2016).
[3] M. Laatiaoui, A. Buchachenko, and L. A. Viehland, Laser Resonance Chromatography of Superheavy Elements, Phys. Rev. Lett. (in press), (2020).

[4] P. R. Kemper and M. T. Bowers, Electronic-state chromatography: application to first-row transition-metal ions, J. Phys. Chem. 95, 5134 (1991). 
[5] W. S. Taylor, E. M. Spicer, and D. F. Barnas, Metastable metal ion production in sputtering dc glow discharge plasmas: characterization by electronic state chromatography, J. Phys. Chem. A 103, 643 (1999).

[6] M. Laatiaoui, H. Backe, D. Habs, P. Kunz, W. Lauth, and M. Sewtz, Low-field mobilities of rare-earth metals, Eur. Phys. J. D 66, 232 (2012).

[7] P. B. Armentrout, Electronic state chromatography, in Ion Mobility Spectrometry-Mass Spectrometry, edited by Sarah Trimpin and Charles L. Wilkins (CRC Press, Taylor \& Francis Group, Boca Raton, London, New York, 2011).

[8] M. J. Manard and P. R. Kemper, Ion mobility mass spectrometry: The design of a new high-resolutionion mobility instrument with applications toward electronic-state characterization of first-row transition metal cations, Int. J. Mass Spectrom. 402, 1 (2016).

[9] M. J. Manard and P. R. Kemper, Characterizing the electronic states of the second-row transitionmetal cations using high-resolution ion mobility mass spectrometry, Int. J. Mass Spectrom. 407, 69 (2016).

[10] C. Iceman, C. Rue, R. M. Moision, B. K. Chatterjee, and P. B. Armentrout, Ion mobility studies of electronically excited states of atomic transition metal cations: Development of an ion mobility source for guided ion beam experiments, J. Am. Soc. Mass Spectrom. 18, 1196 (2007).

[11] A. Kramida, Yu. Ralchenko, J. Reader, and NIST ASD Team (2019), NIST Atomic Spectra Database (ver. 5.7.1) (National Institute of Standards and Technology, Gaithersburg, MD, 2020).

[12] E. V. Kahl, J. C. Berengut, M. Laatiaoui, E. Eliav, and A. Borschevsky, High-precision ab initio calculations of the spectrum of $\mathrm{Lr}^{+}$, Phys. Rev. A 100, 062505 (2019).

[13] J. Brust and A. C. Gallagher, Excitation transfer in barium by collisions with noble gases, Phys. Rev. A 52, 2120 (1995).

[14] R. Loudon, The Quantum Theory of Light (Oxford University Press, Oxford, 2000).

[15] P. Quinet, P. Palmeri, E. Biémont, M. M. McCurdy, G. Rieger, E. H. Pinnington, M. E. Wickliffe, and J. E. Lawler, Experimental and theoretical radiative lifetimes, branching fractions and oscillator strengths in $\mathrm{Lu} \mathrm{II,}$ Mon. Not. R. Astron. Soc. 307, 934 (1999).

[16] E. Paez, K. J. Arnold, E. Hajiyev, S. G. Porsev, V. A. Dzuba, U. I. Safronova, M. S. Safronova, and M. D. Barrett, Atomic properties of $\mathrm{Lu}^{+}$, Phys. Rev. A 93, 042112 (2016).

[17] P. Chhetri et al., Impact of buffer gas quenching on the ${ }^{1} \mathrm{~S}_{0} \rightarrow{ }^{1} \mathrm{P}_{1}$ ground-state atomic transition in nobelium, Eur. Phys. J. D 71, 195 (2017).

[18] A. A. Buchachenko and L. A. Viehland, Mobility of singly-charged lanthanide cations in rare gases: Theoretical assessment of the state specificity, J. Chem. Phys. 140, 114309 (2014).

[19] M. J. Manard and P. R. Kemper, Reduced mobilities of lanthanide cations measured usinghigh-resolution ion mobility mass spectrometry with comparisons between experiment and theory, Int. J. Mass Spectrom. 412, 14 (2017).

[20] A. A. Buchachenko and L. A. Viehland, Ab initio study of the mobility of $\mathrm{Gd}^{+}$ions in $\mathrm{He}$ and $\mathrm{Ar}$ gases, Int. J. Mass Spectrom. 443, 86 (2019).

[21] M. Dolg, H. Stoll, and H. Preuss, Energy-adjusted ab initio pseudopotentials for the rare earth elements, J. Chem.
Phys. 90, 1730 (1989).

[22] X. Cao and M. Dolg, Segmented contraction scheme for small-core lanthanide pseudopotential basis sets, J. Mol. Struct.: THEOCHEM 581, 139 (2002).

[23] A. A. Buchachenko, G. Chałasiński, and M. M. Szczȩśniak, Diffuse basis functions for small-core relativistic pseudopotential basis sets and static dipole polarizabilities of selected lanthanides La, Sm, Eu, Tm and Yb, Struct. Chem. 18, 769 (2007).

[24] D. E. Woon and T. H. Jr. Dunning, Gaussian basis sets for use in correlated molecular calculations. IV. Calculation of static electrical response properties, J. Chem. Phys. 100, 2975 (1994).

[25] S. M. Cybulski and R. R. Toczylowski, Ground state potential energy curves for $\mathrm{He}_{2}, \mathrm{Ne}_{2}, \mathrm{Ar}_{2}, \mathrm{He}-\mathrm{Ne}$, He-Ar, and Ne-Ar: A coupled-cluster study, J. Chem. Phys. 111, 10520 (1999).

[26] P. J. Knowles, C. Hampel, and H.-J. Werner, Coupled cluster theory for high spin, open shell reference wave functions, J. Chem. Phys. 99, 5219 (1993).

[27] C. Knowles, P. J. Hampel and H.-J. Werner, Coupled cluster theory for high spin, open shell reference wave functions (erratum), J. Chem. Phys. 112, 3106 (2000).

[28] H.-J. Werner, P. J. Knowles, G. Knizia, F. R. Manby, M. Schütz, et al., Molpro, version 2015.1, a package of ab initio programs, 2015.

[29] S. F. Boys and F. Bernardi, The calculation of small molecular interactions by the differences of separate total energies. Some procedures with reduced errors, Mol. Phys. 19, 553 (1970).

[30] A. Berning, M. Schweizer, H.-J. Werner, P. J. Knowles, and P. Palmieri, Spin-orbit matrix elements for internally contracted multireference configuration interaction wavefunctions, Mol. Phys. 98, 1823 (2000).

[31] H.-J. Werner and P. J. Knowles, An efficient internally contracted multiconfiguration-reference configuration interaction method, J. Chem. Phys. 89, 5803 (1988).

[32] K. R. Shamasundar, G. Knizia, and H.-J. Werner, A new internally contracted multi-reference configuration interaction method, J. Chem. Phys. 135, 054101 (2011).

[33] H.-J. Werner and P. J. Knowles, A second order multiconfiguration SCF procedure with optimum convergence, J. Chem. Phys. 82, 5053 (1985).

[34] D. Peng and M. Reiher, Exact decoupling of the relativistic Fock operator, Theor. Chem. Acc. 131, 1081 (2012).

[35] Q. Lu and K. A. Peterson, Correlation consistent basis sets for lanthanides: The atoms La-Lu, J. Chem. Phys. 145, 054111 (2016).

[36] L. A. Viehland, Velocity distribution functions and transport coefficients of atomic ions in atomic gases by a Gram-Charlier approach, Chem. Phys. 179, 71 (1994).

[37] L. A. Viehland, Gaseous Ion Mobility, Diffusion, and Reaction (Springer Nature AG, Cham, Switzerland, 2018).

[38] See www.lxcat.net for Plasma Data Exchange Project, 2020.

[39] J. T. Moseley, I. R. Gatland, D. W. Martin, , and E. W. McDaniel, Measurement of transport properties of ions in gases; Results for $\mathrm{K}^{+}$ions in $\mathrm{N}_{2}$, Phys. Rev. A 178, 234 (1969).

[40] O. J. Orient, Ion swarm analysis in electric-field with transport-equation, Acta Phys. Acad. Sci. Hung. 35, 247 (1974).

[41] K. Iinuma, T. Hamano, and M. Takebe, Analysis of two ion swarms in dynamic equilibrium: Simultaneous deter- 
mination of two drift velocities and two reaction frequencies, J. Chem. Phys. 101, 2949 (1994). 